\title{
Sjogern - Larsson Syndrome: A Case Report
}

\author{
RASHIDUL KARIM ${ }^{1}$, HUMAYRA SULTANA ${ }^{2}$, MD. SULTAN UDDIN ${ }^{3}$
}

\section{Introduction}

Sjogren-Larsson Syndrome (SLS) is a rare recessively inherited neurocutaneous disorder with congenital ichthyosis, spastic diplegia or quadriplegia and mental retardation. Additional clinical findings have been reported include characteristic pruritus, retinopathy, preterm birth and skeletal abnormality. ${ }^{1} \mathrm{SLS}$ is an inborn error of lipid metabolism caused by a deficiency of the microsomal enzyme fatty aldehyde dehydrogenase (FALDH), a component of fatty alcohol: NADOxidoreductase enzyme complex. This enzyme catalyzes the oxidation of long chain fatty aldehydes to fatty acids. Accumulation of long chain fatty alcohols and modification of macromolecules by an excess of fatty aldehydes are thought to be the pathophysiologic mechanisms causing the manifestation of SLS. ${ }^{2}$ In 1994, the FALDH gene was mapped to chromosome 17p11.2. Mutation analysis has identified many different mutations in the FALDH gene in SLS patients. ${ }^{3}$ The disease is most common in Sweden, where an estimated 0.4 out of 100,000 are affected by it. Outside Sweden prevalence is unknown. Over 200 cases worldwide have been reported. ${ }^{4}$ Here we are reporting one case in a Bangladeshi family.

\section{Case Report}

A $2 \frac{1}{2}$ year-male child from Bangladeshi family, hailed from old Dhaka city was admitted in the pediatric department of Dhaka National Medical College and Hospital (DNMCH) with complaints of fever, oral ulcer, spasticity of both lower and upper limbs, and also generalized dry, scaly skin with severe pruritus. The patient was the first issue of consanguinous parents who were healthy. He was preterm. There was history of recurrent convulsion from $11 / 2$ years of age. Child attained head control at 6 months, sitting without support at 10 months of age. Spasticity in lower limbs started in later part of the first year of life with progressive increase. Neurological examination revealed increased tone in both upper and lower limbs,

1. Associate Professor of Paediatrics, Dhaka National Medical College and Hospital, Dhaka, Bangladesh.

2. Assistant Professor of Paediatrics, Dhaka National Medical College and Hospital, Dhaka, Bangladesh.

3. Professor of Paediatrics, Dhaka National Medical College and Hospital, Dhaka, Bangladesh.

Correspondence: Dr. Rashidul Karim. Email: rashidul34@ gmail.com brisk deep tendon reflexes and bilateral extensor plantar response suggestive of spastic quadriplegia, and mental retardation. There was also evidence of conduction aphasia in the child. Child had a normal head circumference for his age. Eye examination showed white dots on retina and photophobia. Skin examination showed scaly ichthyotic lesions with severe pruritus presently affecting all body parts. It started since birth on the face.

All routines hematological investigations and chest radiograph were normal. His Electroencephalography (EEG) demonstrates multifocal epileptic discharges (e.g. spike and wave complexes, polyspikes, sharp waves of high voltage) occurring in the presence of disorganized background activity. Computed tomography (CT) scan revealed hypodense areas in the periventricular white matter. Skin biopsy demonstrated lamellar ichthyosis. These finding were compatible with SLS. All these clinical and radiological findings were diagnostic of Sjogren-Larsson syndrome (SLS).

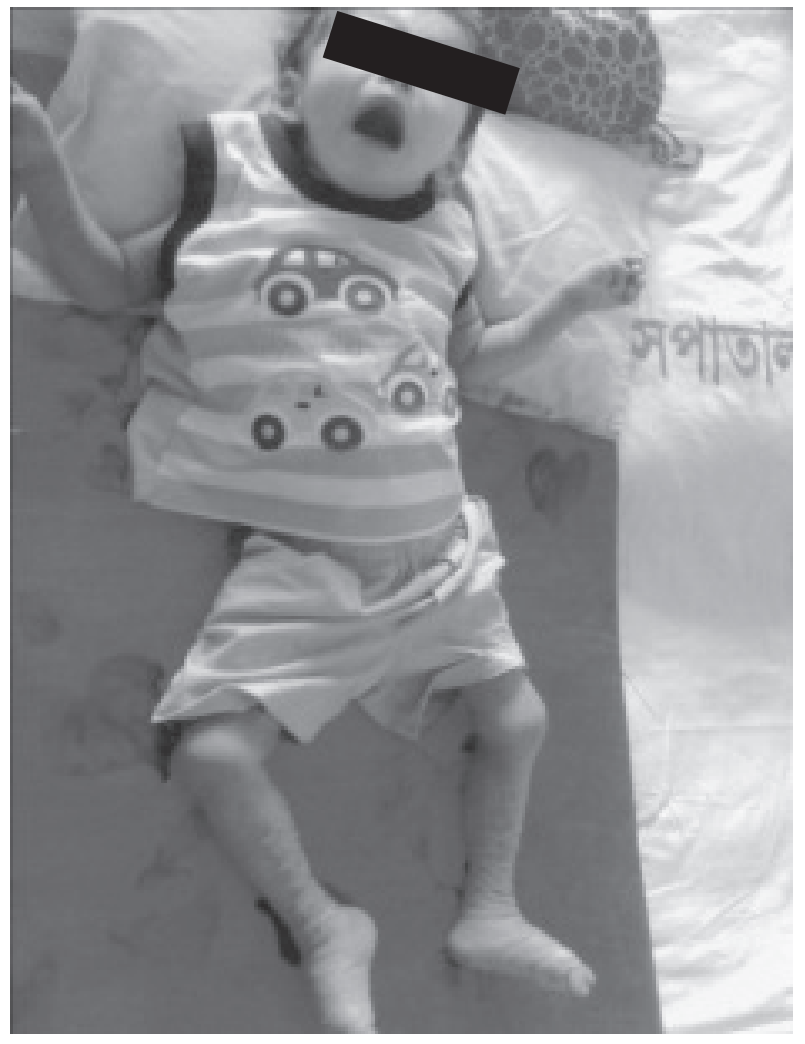

Fig-1: Generalized dry scaly skin of the patient 


\section{Discussion}

Sjogren Larsson Syndrome (SLS) is a rare inherited disease characterized by inability of the body to breakdown certain molecules called fatty aldehydes and fatty alcohols due to a deficiency in the activity of an enzyme called fatty aldehyde dehydrogenate (FALDH). Symptoms associated with SLS are attributed to the accumulation of fatty aldehydes and fatty alcohols in various tissues in the body. ${ }^{5}$

In 1957, Sjogren and Larsson described a rare syndrome consisting of congenital ichthyosis associated with spastic diplegia or quadriplegia (spastic pyramidal syndrome), aphasia and low grade dementia. ${ }^{6}$ SLS can occur in individuals of all races and ethnicities, but is most common in individuals of Swedish ancestry. In Sweden, the annual incidence of SLS is approximately 1 in 167,000 and the carrier frequency is approximately 1 in $200 .^{7}$ In our country this rare disease is not yet reported.

Clinical features develop prenatally and during infancy. There is usually spastic diplegia, occasionally tetraplegia/ quadriplegia, with mental retardation, epilepsy, speech defects, dental, dermatological, skeletal and retinal changes. ${ }^{8}$ Skin changes are in form of ichthyosis which is a generalized hyperkeratosis (skin become dry, rough and scaly with a brownish or yellowish tone). These skin abnormalities are generally dispersed over the whole body, most severely affecting the flexural areas, the nape of the neck, the trunk and the extremities. Mild hyperkeratosis is usually present at birth and progresses to a generalized ichthyosis during the first year of life. Pruritus is a prominent feature that is not found in other types of ichthyotic skin disorders. ${ }^{3}$ This finding was present in our case.

The diagnosis of SLS is almost always delayed because usually only cutaneous symptoms are present at birth. Newborns usually manifest symptoms and signs of the disease (first ichthyosis, subsequently neurologic symptoms). Since the skin disorder is the most prominent sign in infancy, patient was first seen by dermatologist and minor neurologic signs were missed. The diagnosis was made later when neurologic signs appeared. ${ }^{4}$ Neurologic signs usually appear between 4 and 30 months of age and the main neurological features are spastic diplegia or quadriplegia, developmental delays, mental retardation and conduction aphasia. About $30-60 \%$ of patients have convulsion during infancy. ${ }^{8}$ In our case all above neurological findings were present. Neuropathologically, the hallmark SLS is demyelination of the cerebral white matter and corticospinal and vestibulospinal tract which causes spasticity. ${ }^{9}$ These abnormalities are due to accumulation of lipids and fatty alcohol in the myelin as a consequence of an enzymatic defect. ${ }^{3}$ The diagnosis of SLS should be considered in all patients with generalized hyperkeratosis and central nervous system dysfunction. The presence of glistening dots in the macular region is considered pathognomonic, although it is not constant. The nature of the crystalline deposits in the retina is unclear. It is speculated that they might represent accumulations of long-chain fatty alcohols or fatty aldehydes. ${ }^{10}$ This finding was present in this case. Few reports have described CT and MRI finding of SLS. CT scan revealed hypodense areas in the perivestricular white matter. ${ }^{8} \mathrm{MR}$ imaging shows retardation of myelination and dysmyelination. In SLS, MR imaging abnormalities of the brain are confined to the cerebral white matter and the corticospinal tracts. They consist of the accumulation of lipid substrates, delayed myelination, periventricular gliosis, and a permanent myelin deficit. ${ }^{4}$ Skin biopsy demonstrated a lamellar ichthyosis. ${ }^{8}$ This finding was present in this case. The diagnosis of SLS was confirmed by demonstration of the enzyme defect in cultured skin fibroblast can provide biochemical confirmation ${ }^{3}$. We could not perform these because of equipment limitation. Though in such cases biochemical (Urinary concentration of leucotriene B4 and 20-OH-LTB4 and genetic studies (for ALDH3A2 gene) are desired we could not perform these due to paucity of resources. SLS can be diagnosed prenatally using enzymatic methods by measuring FAO (Fatty alcohol: NAD+ Oxidoreductose) and FALDH (fatty aldehyde dehydrogenase) in cultured amniocytes or cultured chorionic villus cells. ${ }^{11}$

Management is SLS is supportive, because effective casual therapy strategies are not available to date; however, gene therapy studies may be successful in future. Treatments for the skin problems caused by ichthyosis include daily bath, topical moisturizing lotion, keratolytics agents and oral retinoid. Training to provide home-based physical therapy is useful to prevent contractures. Surgery may help to reduce the spastic movements of the leg. If a person with SLS has seizures, these usually can be controlled with anti-seizure medication. ${ }^{4}$ So management should be 
multidisciplinary with input from physiotherapy, occupational therapy, dermatology, speech therapy, neurology, ophthalmology and orthopedic services.

\section{Conclusion}

Early diagnosis and multidisciplinary management protocol may lead a better life of the child and minimize the sequelae.

\section{References}

1. Willemsen MA, Rotteveel JJ, Dombarg PH, Gabreels FJ, Mayatepek E, Sengers RC. Preterm birth in Sjogren-Larsson Syndrome. Neuropediatrics 1999; 30: 325-27.

2. Haugs, Braun-Falco M. Restoration of fatty aldehyde dehydrogenase deficiency in SjogrenLarsson Syndrome.Gene Ther 2006; 13: 1021-26.

3. Willemsen MA, ljlst L, Steijlen PM, Rotteveel JJ, Jong JanGN, VanDomburg PHM, et al. Clinical, Biochemical and molecular genetic characteristics of 19 patients with the SLS. Brain 2001;124:1426-37.

4. Moghaddam FR, Safar F, Asheghan M, Soltani ZR, Zade FD. Sjogren-Larsson Syndrome in two brothers: a case report. Cases J 2009; 2: 84.

5. Rizzo WB, Carney G, Lin Z. The molecular Basis Sjogren-Larsson Syndrome: Mutation Analysis of the Fatty aldehyde Dehydrogenase Gene. Amj.Hum Genet 1999; 65: 1547-60.

6. Sjogren T, Larsson T. Oligophrenia in combination with congenital ichthyosis and spastic disorders. Acta Psychiatr scand 1957; 32: 1-108.

7. Jagell S, Gustavson KH, Holmgren G. SjogrenLarsson Syndrome in Sweden: a clinical, genetic and epidemiological study. Clin Genet 1981; 19:233-56.

8. Dutra LA, De Acquino CC, Barsottini OG. Sjogren-Larsson Syndrome: Case report and review of neurologic abnormalities and ichthyosis. Neurologist 2009; 15: 332-34.

9. Wester $P$, Bergstrom $U$, Brun $A$, Jagell $S$, Karisson B, Eriksson A. Monaaminergic dysfunction in Sjogren-Larsson Syndrome. Molchem Neuropathol 1991; 15: 13-28.

10. Willemsen MA, Cruysberg JR, Rotteveel JJ, Aandekerk AL, Van Domburg PH, Dentman AF. Juvenile macular dystrophy associated with deficient activity of fatty aldehyde dehydrogenase in Sjogren-Larsson Syndrome. Am J ophthalmol 2000; 130: 782-89.

11. Rizzo WB, Craft DA, Kelson TL, Bonnefont JP, Saudubray JM, Schulman JD et al. Prenatal diagnosis of Sjogren-Larsson Syndrome using enzymatic methods. Prenatal Diagnosis 1994; 14: 577-81. 